\title{
IMPROVEMENTS TO THE FERMILAB IONIZATION PROFILE MONITOR SYSTEMS
}

\author{
J. R. Zagel, A. A. Hahn, J. L. Crisp, C. Jensen, FNAL, Batavia, IL
}

\begin{abstract}
The Ion Profile Monitor Systems have been studied in the Fermilab Tevatron, Main Injector, and Booster accelerators. These systems capture $64 \mathrm{~K}$ samples of both horizontal and vertical profiles at a turn by turn sample rate. Some early results have revealed various systematic problems and where improvements in the present system can be accomplished. Identification of these systematics and improvements for these systems are described. An entirely new design is planned which incorporates a magnetic field and can collect electrons instead of ions. In addition, selective gating of the top microchannel plate in a 2-plate system will allow us to minimize saturation and charge depletion problems.
\end{abstract}

\section{CURRENT INSTALLATIONS}

Ionization Profile Monitors (IPM) are installed in the Fermilab Booster [1] and Main Injector. Two devices that were previously installed in the Main Ring [2] were removed, new microchannel plate (MCP) glass installed, and then placed in the new Main Injector.

The Booster system has an $8 \mathrm{kV}$ clearing field and currently collects positive ions. The reworked Main Injector devices operate at $28 \mathrm{kV}$, also collecting positive ions.

\section{SATURATION}

The system is timed to observe about $1 \mu \mathrm{sec}$ of beam at a set time delay from each revolution marker. This time corresponds to just under 1 Booster batch. We would expect that the signal observed should be directly proportional to the number of ions collected. It was recognized that the area of the profile should be consistent throughout the acceleration cycle and proportional to the ionization produced by the fraction of beam current within our $1 \mu \mathrm{sec}$ integration time. [3] Area is defined as the sigma multiplied by the amplitude from a gaussian fit. When motion of the beam caused a change in position on the MCP, ions impinging on a different area of the MCP caused greater amplitude peaks for some number of turns. When additional batches are injected, a corresponding loss of signal occurs that is not consistent with any losses noted from the observed batch. Instead a much larger loss is noted in the beam-induced signal. An oscilloscope trace taken during a short run in the Tevatron also showed this behavior.

* Work supported by the U.S. Department of Energy under Contract No.DE-AC02-76CH03000

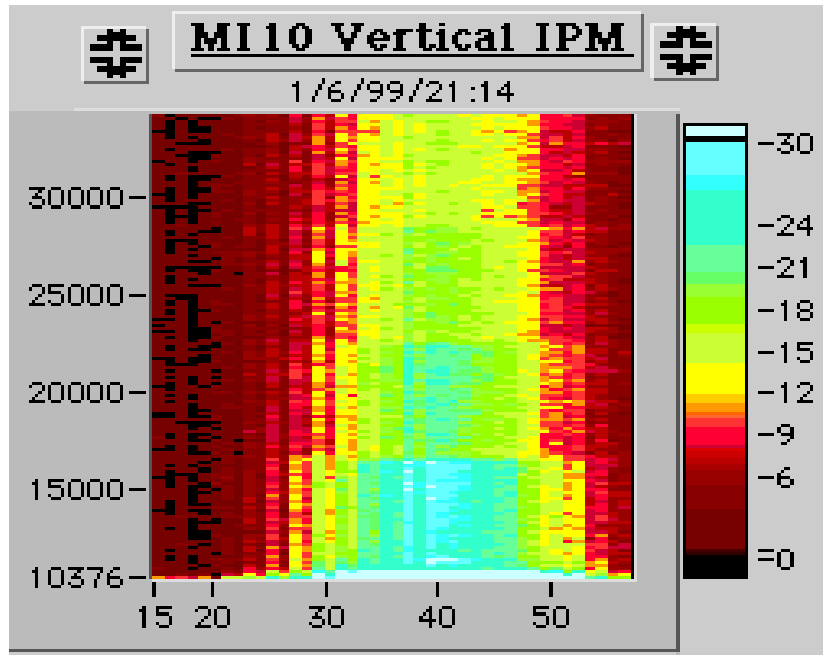

Figure 1. Injection effects on observed batch.

Figure 1 shows the intensity profile for the time period during which 4 Booster batches are injected into Main Injector. The intensity decrease noted after each batch is injected, approximately 6000 samples, is the result of charge depletion from the MCP not beam losses in the observed batch.

Three potential solutions to this problem are: 1 . obtain an MCP with greater output current capability, 2. higher gain preamplifiers, or 3 . use the dual chevron MCP configuration in a mode where the ion collection plate is used as a fast gate while the lower plate is used to provide gain. This mode of operation allows the signal producing plate to recharge during the off time of the gating plate.

\subsection{High Output MicroChannel Plates}

The MCP's used in these systems originate from Galileo Corporation. Extended Dynamic Range (EDR) glass is available that can provide a 4 to 10 times improvement in output current. Typically an MCP can provide 10 to $20 \%$ of the bias current as a linear output.

Table 1: MicroChannel Plate specifications

\begin{tabular}{|l|c|c|}
\hline $\begin{array}{c}\text { MCP } \\
\text { Type }\end{array}$ & $\begin{array}{c}\text { Bias Current } \\
\mathbf{1 0 0 0} \text { Volts }\end{array}$ & Gain \\
\hline Long Life & $120 \mu \mathrm{amps}$ & $2.5^{*} 10^{4}$ \\
\hline EDR & $420 \mu \mathrm{amps}$ & $2.6^{*} 10^{4}$ \\
\hline
\end{tabular}

We have been using simple voltage dividers to provide bias voltages for the existing dual chevron plate assemblies. This will not provide the appropriate bias using the newer extended dynamic range glass, which 
exhibits much lower impedance. Dual power supplies will be used to provide a separately adjustable bias for each plate. The top plate will then be pulsed to provide a gate consistent with the batch to be observed. For the Fermilab Main Injector the gate will be on for $1.6 \mu \mathrm{sec}$ out of an approximately $11.1 \mu \mathrm{sec}$ revolution period.

\subsection{Gating McroChannel Plates}

To provide time gating of the micro channel plate, the first stage must be gated by applying a voltage pulse during the time of interest. The ultimate goal is to have this stage go from below threshold to above threshold in approximately $50 \mathrm{nsec}$ (3 empty bunches between batches). The application requires a series of $1.6 \mu \mathrm{sec}$ pulses to repeat for approximately 1 second and then have 0.5 seconds of off time. There are two approaches, each requiring a fast pulse generator and an isolation transformer: 1) keep the plate biased at below nominal operating voltage and then supply a $200 \mathrm{~V}$ pulse, or 2) keep the plate at $0 \mathrm{~V}$ and apply the full necessary voltage.

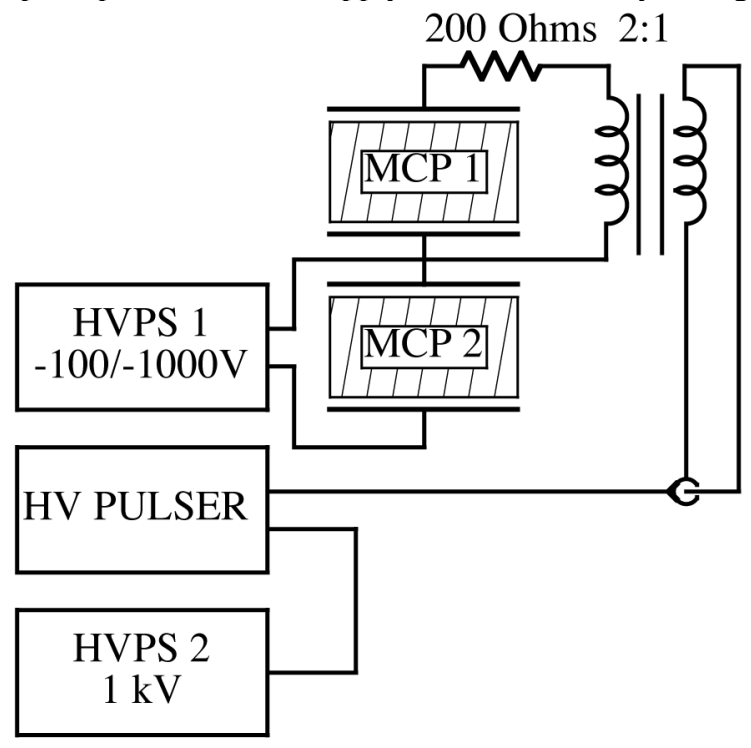

Figure 2: MCP Bias scheme.

The kicker group at Fermilab has developed a fast high voltage pulse generator for their work [4]. This can be used to generate a pulse of up to $800 \mathrm{~V}$ with a rise time of $15 \mathrm{nsec}$ into $50 \mathrm{Ohms}$. An additional power supply is needed to remotely control either the variable bias or to supply the variable pulse amplitude from the pulse generator. There is a tradeoff in how much power is dissipated in resistors. For the biased case, a DC blocking capacitor is required but this must be discharged otherwise the pulse current would overcharge it. For the full voltage case, the termination resistor will have a much higher power dissipation. The latter solution requires fewer components, and failures in the circuit tend to inhibit operation of the MCP instead of causing damage. There will be only passive components mounted in the tunnel, external to the vacuum system, connected via several hundred feet of $50 \Omega$ cable.

\section{SIGNAL CONDITIONING}

When the Main Injector started to ramp we observed a potential difference in tunnel ground as referenced to the service building. The IPM has only a few microseconds to measure nanoampers of current. Even small differences in ground can cause problems.

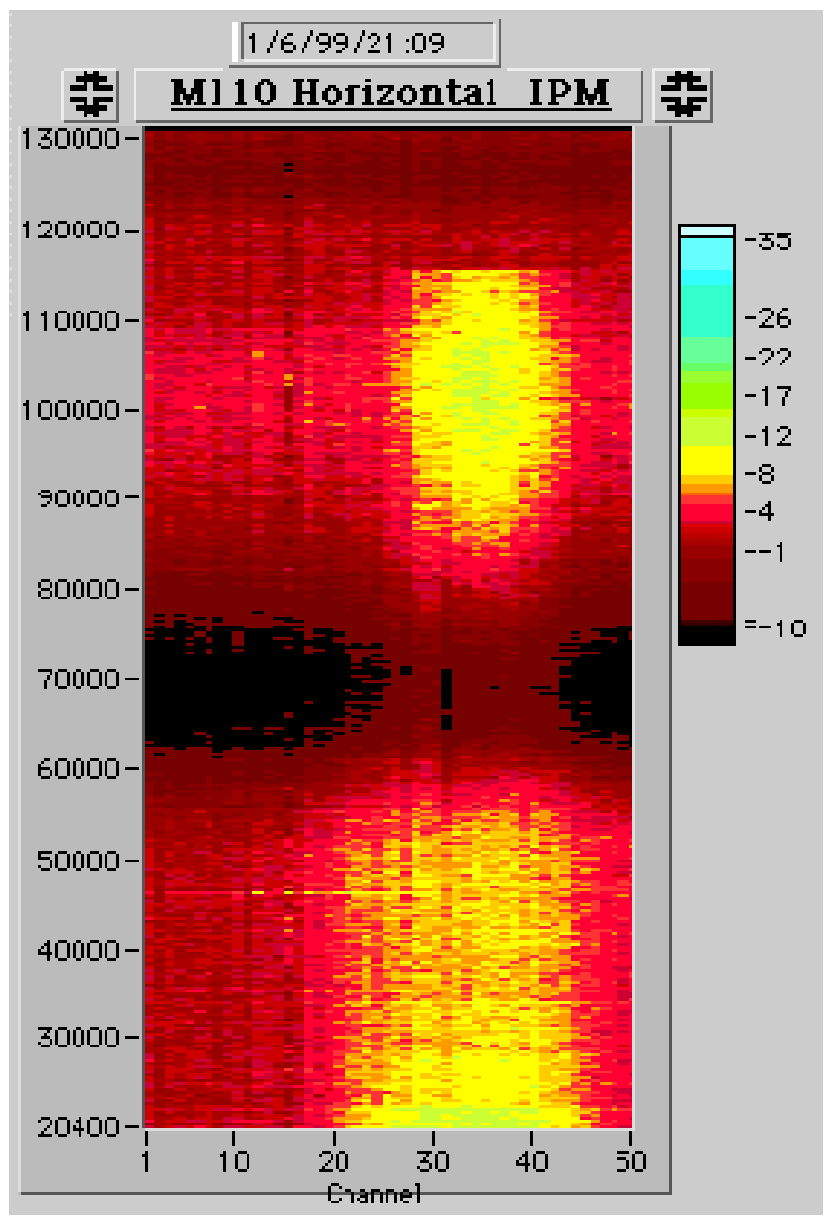

Figure 3: Signal variations through acceleration cycle.

The apparent loss of signal in figure 3, sample 62000 through 77000, is explained by the capacitive coupling of ramp currents into the beam pipe ground. Since the system software calculates a baseline in the fitting routines this does not affect the final profile information. However steps have been taken to capacitively decouple the grounds to prevent possible damage to the amplifiers.

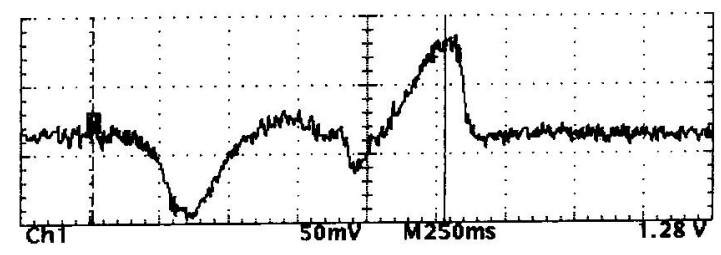

Figure 4: Shorted RG-58 signal as seen from upstairs. 
The capacitance to ground in the main bend magnets allows bend bus voltage to induce currents along the beam pipe. As a power supply ramps up, the beam pipe closes the loop between capacitive coupling to ground through the magnets on both sides of the supply. The IPM's in the Main Injector are located 17 meters apart and a power supply feeds the bend bus half way between them. The measured ground potential between IPM's peaks at 150 millivolts and is proportional to $\mathrm{d} / / \mathrm{dt}$ in the bend buss. Ohms law suggests 5 amps of current flows through the $.0017 \Omega / \mathrm{m}$ stainless beam pipe separating the IPM's.

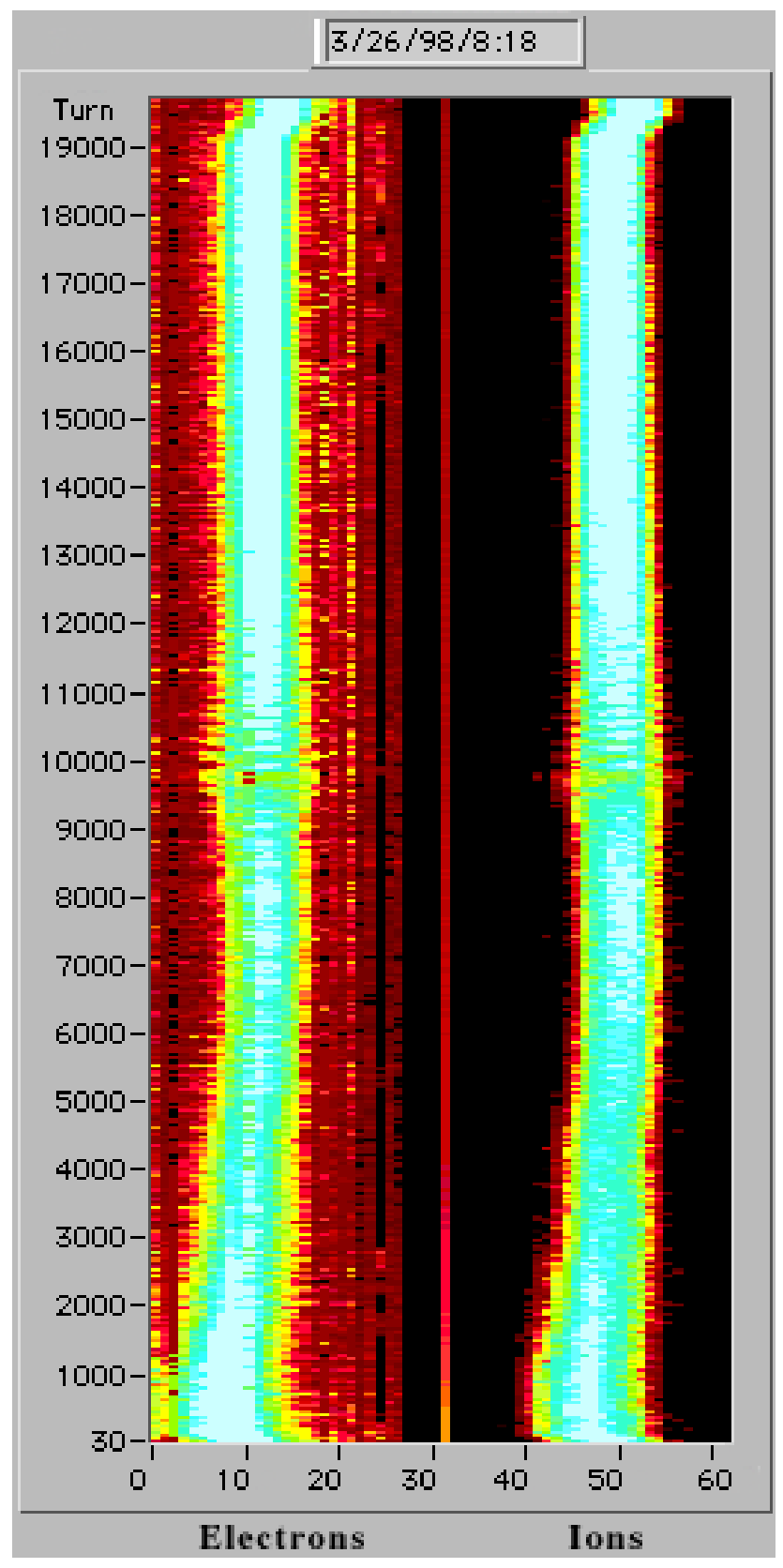

Figure 5: Electron collection test in Booster at 600 gauss.

\section{THE CASE FOR ELECTRON COLLECTION}

The collection of positive ions produced in the proton beam is problematic in that the space charge of the beam itself contributes to spreading those ions. The problem is exacerbated by the smaller beam sizes, in the Main Injector $(1 \mathrm{~mm})$ and Tevatron $(.5 \mathrm{~mm})$, where the spreading increases the measured profiles by up to $500 \%$ over the actual beam size. To eliminate this effect we are adding a magnetic field and switching the polarity of the clearing field to collect electrons. An experiment was done in the Fermilab Booster to determine the effects of placing a magnetic field around the IPM and collection of electrons. The conclusion is that electron collection is at least as good as with positive ions. In the Booster the expected ion spreading was at the $10 \%$ level at most. We were dominated by systematic effects due to saturation at these signal levels and could not decisively prove that the electron profiles were $10 \%$ smaller than the ion profiles.

Calculations suggest that a magnetic field of 2000 Gauss for the Main Injector and 3000 Gauss for the Tevatron will be sufficient to contain electrons produced in the beam while they are accelerated toward the MCP. This field will reduce spreading to the 1 to $2 \%$ level.

\section{WORK IN PROGRESS}

High output glass has been installed into the Main Injector IPM's. We are now building the additional power pulsing devices to install for the next run. By using the top plate of the chevron configuration as a gate and the bottom plate for gain we hope to minimize the effects of saturation on the lower plate.

Design work is now being undertaken to produce a magnet and improved mounting scheme for the internal devices to allow us to capture electrons on the MCP's in the Main Injector and Tevatron Installations.

\section{REFERENCES}

[1] Zagel, J. R., Chen, D., Crisp, J. L., "Fermilab Booster Ion Profile Monitor System Using LabVIEW",

1994 Beam Instrumentation Workshop,

AIP Conference Proceedings 333, pp384-390.

[2] J. R. Zagel, J. L. Crisp, A. H. Hahn, P.G. Hurh,

"Main Ring Ion Profile Monitor System",

1997 Particle Accelerator Conference, IEEE Conference Proceedings 97CH36167, pp2166-2168.

[3] Alan A. Hahn, and James R. Zagel, "Observation of Bethe-Bloch Ionization using the Booster Ion Profile Monitor", Paper THAR4 of this conference.

[4] C. Jensen, D. Qunell, "A Thyratron Trigger with Low Jitter", Proceedings of 1997 Particle Accelerator Conference, IEEE Conference Proceedings 97CH36167, pp1281-1283. 